\title{
Degree Distribution and Number of Edges between Nodes of Given Degrees in the Buckley-Osthus Model of a Random Web Graph
}

\author{
Evgeniy A. Grechnikov
}

Abstract. In this paper, we study some important statistics of the random graph $H_{a, k}^{(t)}$ in the Buckley-Osthus model, where $t$ is the number of nodes, $k t$ is the number of edges (so that $k \in \mathbb{N}$ ), and $a>0$ is the so-called initial attractiveness of a node. This model is a modification of the well-known Bollobás-Riordan model. First, we find a new asymptotic formula for the expectation of the number $R(d, t)$ of nodes of a given degree $d$ in a graph in this model. Such a formula is known for $a \in \mathbb{N}$ and $d \leq t^{1 / 100(a+1)}$. Both restrictions are unsatisfactory from theoretical and practical points of view. We completely remove them. Then we calculate the covariances between any two quantities $R\left(d_{1}, t\right)$ and $R\left(d_{2}, t\right)$, and using the second-moment method we show that $R(d, t)$ is tightly concentrated around its mean for all possible values of $d$ and $t$. Furthermore, we study a more complicated statistic of the web graph: $X\left(d_{1}, d_{2}, t\right)$ is the total number of edges between nodes whose degrees are equal to $d_{1}$ and $d_{2}$ respectively. We also find an asymptotic formula for the expectation of $X\left(d_{1}, d_{2}, t\right)$ and prove a tight concentration result. Again, we do not impose any substantial restrictions on the values of $d_{1}, d_{2}$, and $t$. 


\section{Introduction}

The real world has many interesting structures that can be thought of as graphs. A typical example is the World Wide Web: one can consider web pages to be nodes of a graph and hyperlinks to be edges. One of the productive methods for studying these graphs involves investigation of a suitable random graph model.

The first models of random graphs were constructed and investigated long ago. Classical models and results are systematized, for example, in [Bollobás 01] and [Janson et al. 00]. However, they are not suitable for approximation of dynamically changing and nonuniform networks. In particular, the degree sequences of the graphs in these models are very far from those observed in reality.

Recently, other models of random graphs were constructed to more closely match the growth of real networks. One of the first descriptions of such a model can be found in the article [Barabási and Albert 99], in which the authors introduced the "preferential attachment" rule. Models following this rule assign the probability of a new edge to a node according to the current degree of that node, so more "popular" nodes are more attractive for new edges.

However, the article [Barabási and Albert 99] did not contain a precise model, leaving some parameters unspecified. Variations of these parameters can significantly change properties of graphs that arise, as shown in [Bollobás and Riordan 03], so one needs something more explicit for theoretical investigations. An explicit model was proposed in [Bollobás et al. 01] based on the preferential attachment rule. In the same article, the authors rigorously proved a theorem concerning the degree sequence of a graph in this model. Namely, they showed that the number of nodes with degree $d$ in their model decreases proportionally to $d^{-3}$. The same quantity in real networks decreases proportionally to $d^{-\gamma}$ with different $\gamma$ for different networks, following the so-called power law.

The Bollobás-Riordan model has only one parameter, a natural number representing the ratio of the number of edges to the number of nodes. Thus, on the one hand, the Bollobás-Riordan model certainly matches some real networks by explaining the power law. But on the other hand, the number of parameters in this model is small and does not allow one to obtain the power law with an exponent that is not equal to -3 .

In the Bollobás-Riordan model, the probability that a node is a target for a new edge is proportional to the degree of that node. In [Dorogovtsev et al. 00] and [Drinea et al. 01], two groups of researchers independently proposed adding to the model one more parameter - an "initial attractiveness" of a node, which is a positive constant not depending on the degree. Equivalently, the probability in the proposed model is a linear function of the degree. However, in the papers 
[Dorogovtsev et al. 00] and [Drinea et al. 01], we find only some heuristic arguments.

In [Buckley and Osthus 04], the authors gave an explicit construction of the above-described model and rigorously proved a theorem concerning the degree sequence of a graph in this model when all the parameters are natural numbers.

Among many articles in this area, we also quote [Jordan 06]. The model investigated in that article differs from the Buckley-Osthus model, but the difference is small, so the results are comparable. The article deals with the case in which parameters are not necessarily natural numbers. However, the proven theorem works only for fixed degree $d$ as the number of nodes tends to infinity; Bollobás et al. as well as Buckley and Osthus allowed $d$ to grow as some small power of the number of nodes.

There are many other random graph models intended to approximate real networks. We refer the reader to [Bollobás and Riordan 03] and [Durrett 07] for surveys of such models and corresponding results.

We study the Buckley-Osthus model of a random graph. Our first goal is to give a significant improvement of the above-mentioned theorem from [Buckley and Osthus 04] using a completely different method. We find an asymptotic formula for the expectation of the number of nodes with degree $d$ without any upper bound on $d$ and with an estimate of the error term. We also prove a tight concentration result.

Since the Bollobás-Riordan model is a special case of the Buckley-Osthus model, our results are also applicable to it. So again, we get a substantial improvement of the main theorem from [Bollobás et al. 01].

Our second goal is to study the following quantity. We fix two numbers $d_{1}$ and $d_{2}$. We consider a node with degree $d_{1}$ and a node with degree $d_{2}$. Then we calculate the number of edges between these nodes. When there are several choices for nodes of given degrees, we calculate the mean value. Since the number of nodes with a fixed degree is known to have tight concentration around its expectation, it is sufficient to examine the total number of edges linking a node with degree $d_{1}$ and a node with degree $d_{2}$. Here we also obtain an asymptotic formula for the expectation and prove a tight concentration result.

\section{The Model and Formulation of Results}

The Buckley-Osthus model has two parameters, a natural number $k$ and a positive real number $a$. The number $k$ is the ratio of the number of edges to the number of nodes. We assume that $a$ and $k$ are constants, so by default all other 
constants may depend on them. The Bollobás-Riordan model is a special case of this model with $a=1$.

The model is defined in two stages. At the first stage, a probability space $H_{a, 1}^{(t)}$ is constructed. The elements of $H_{a, 1}^{(t)}$ are undirected graphs with nodes represented by numbers $1, \ldots, t$ and with $t$ edges. The space $H_{a, 1}^{(1)}$ contains only one graph with one node and one loop. Every graph in $H_{a, 1}^{(t)}$ is obtained from a graph in $H_{a, 1}^{(t-1)}$ by adding a new node $t$ and a new edge between $t$ and a node $\gamma \in\{1, \ldots, t\}$, so that

$$
\operatorname{Pr}(\gamma=s)= \begin{cases}\frac{\operatorname{deg}_{t-1}(s)-1+a}{(a+1) t-1}, & 1 \leq s \leq t-1, \\ \frac{a}{(a+1) t-1}, & s=t,\end{cases}
$$

where $\operatorname{deg}_{t-1}$ denotes the degree of a node in the graph from $H_{a, 1}^{(t-1)}$. At the second stage, a final probability space $H_{a, k}^{(t)}$ is constructed from $H_{a, 1}^{(t k)}$ as follows. We take any graph from $H_{a, 1}^{(t k)}$. It has $k t$ nodes and $k t$ edges. We identify the nodes $1, \ldots, k ; k+1, \ldots, 2 k ; \ldots$, obtaining $t$ new nodes, and we keep all the edges, obtaining multiple edges and even multiple loops.

We study the number of nodes of degree $d$ in $H_{a, k}^{(t)}$ as a function of $d$ and $t$. We denote this random quantity by $R(d, t)$ and the value of its expectation by $r(d, t)=E R(d, t)$.

If $d<k$, then clearly $R(d, t)=0$, so it suffices to study the case $d \geq k$. We start by considering $r(d, t)$.

Theorem 2.I. Let $d \geq k$. The expected value of $R(d, t)$ is

$$
r(d, t)=\frac{\mathrm{B}(d-k+k a, a+2)}{\mathrm{B}(k a, a+1)} t+O_{a, k}\left(\frac{1}{d}\right) .
$$

The asymptotic behavior of the coefficient as $d$ grows is

$$
\frac{\mathrm{B}(d-k+k a, a+2)}{\mathrm{B}(k a, a+1)} \sim \frac{\Gamma(a+2)}{\mathrm{B}(k a, a+1)} d^{-2-a}=(a+1) \frac{\Gamma(k a+a+1)}{\Gamma(k a)} d^{-2-a} .
$$

A similar result was obtained in [Buckley and Osthus 04] (with some factorials instead of gamma and beta functions). However, for that result, it was essential that $a \in \mathbb{N}$ and $d \leq t^{1 / 100(a+1)}$. Another result, which can be compared with that of Theorem 2.1, is proved in [Jordan 06]. It concerns a slightly different model, but nevertheless, it is rather close to our investigations. In this result, $a$ can be any positive real number (and so analogous gamma and beta functions appear in its statement). However, its proof essentially uses the assumption that $d$ is 
just a constant. In our Theorem 2.1, we do not have any restrictions on $d$ and $a$, and we use a completely different method to prove it.

In fact, Theorem 2.1 gives an entire picture of what happens to the quantity $r(d, t)$. If $d=o\left(t^{\frac{1}{a+1}}\right)$, then Theorem 2.1 yields the main term of $r(d, t)$. If $d=\Omega\left(t^{\frac{1}{a+1}}\right)$, then $r(d, t)$ tends to zero as $t \rightarrow \infty$, which means that with high probability there are no nodes of degree $d$ in a graph in the model.

Now we want to study in detail the quantity $R(d, t)$.

Theorem 2.2. Let $d_{1} \geq k$ and $d_{2} \geq k$. The covariance between $R\left(d_{1}, t\right)$ and $R\left(d_{2}, t\right)$ is

$$
\operatorname{cov}\left(R\left(d_{1}, t\right), R\left(d_{2}, t\right)\right)=O_{a, k}\left(\left(d_{1}^{-2-a}+d_{2}^{-2-a}\right) t+d_{1}^{-1} d_{2}^{-1}\right)
$$

Substituting $d_{1}=d_{2}=d$ in Theorem 2.2 and using Chebyshev's inequality, we obtain the following result.

Corollary 2.3. If $d=d(t) \geq k$ and $\psi(t) \rightarrow \infty$ as $t \rightarrow \infty$, then

$$
\left|R(d, t)-\frac{\mathrm{B}(d-k+k a, a+2)}{\mathrm{B}(k a, a+1)} t\right| \leq\left(\sqrt{d^{-a-2} t}+d^{-1}\right) \psi(t)
$$

with probability tending to 1 as $t \rightarrow \infty$.

Let us discuss the meaning of Corollary 2.3.

When $d \sim C t^{\frac{1}{a+2}}$ with some constant $C$, both $r(d, t)$ and $\sqrt{d^{-a-2} t}+d^{-1}$ are $O(1)$. For smaller values of $d$ (i.e., $d=o\left(t^{\frac{1}{a+2}}\right)$ ), inequality (2.1) implies the equivalence (with probability tending to 1 as $d, t \rightarrow \infty$ )

$$
R(d, t) \sim \frac{(a+1) \Gamma(k a+a+1)}{\Gamma(k a)} d^{-2-a} t .
$$

For larger values of $d$ (i.e., $t^{\frac{1}{a+2}}=o(d)$ ), inequality $(2.1)$ means that $R(d, t)=$ $o(1)$. Since $R(d, t)$ is an integer by definition, we have $R(d, t)=0$ (again with probability tending to 1 as $d, t \rightarrow \infty)$. Thus, we have an almost entire picture of what happens to $R(d, t)$.

We also study the total number of edges linking a node with degree $d_{1}$ and a node with degree $d_{2}$. We denote this random quantity by $X\left(d_{1}, d_{2}, t\right)$. When $d_{1}=d_{2}$, we count every edge twice, but do not count loops.

Theorem 2.4. Let $d_{1} \geq k$ and $d_{2} \geq k$. There exists a function $c_{X}\left(d_{1}, d_{2}\right)$ such that

$$
E X\left(d_{1}, d_{2}, t\right)=c_{X}\left(d_{1}, d_{2}\right) t+O_{a, k}(1)
$$


and

$$
\begin{aligned}
& c_{X}\left(d_{1}, d_{2}\right)=\frac{\Gamma\left(d_{1}-k+k a\right) \Gamma\left(d_{2}-k+k a\right) \Gamma\left(d_{1}+d_{2}-2 k+2 k a+3\right)}{\Gamma\left(d_{1}-k+k a+2\right) \Gamma\left(d_{2}-k+k a+2\right) \Gamma\left(d_{1}+d_{2}-2 k+2 k a+a+2\right)} \\
& \quad \times k a(a+1) \frac{\Gamma(k a+a+1)}{\Gamma(k a)} \\
& \quad \times\left(1+\theta\left(d_{1}, d_{2}\right) \frac{\left(d_{1}-k+k a+1\right)\left(d_{2}-k+k a+1\right)}{\left(d_{1}+d_{2}-2 k+2 k a+1\right)\left(d_{1}+d_{2}-2 k+2 k a+2\right)}\right),
\end{aligned}
$$

where

$$
-4+\frac{2}{1+k a} \leq \theta\left(d_{1}, d_{2}\right) \leq a \frac{\Gamma(k a+1) \Gamma(2 k a+a+3)}{\Gamma(2 k a+2) \Gamma(k a+a+2)} .
$$

As both $d_{1}$ and $d_{2}$ grow, the asymptotic behavior of $c_{X}$ is

$$
\begin{aligned}
& c_{X}\left(d_{1}, d_{2}\right)=k(a+1) \frac{\Gamma(k a+a+1)}{\Gamma(k a)} \frac{\left(d_{1}+d_{2}\right)^{1-a}}{d_{1}^{2} d_{2}^{2}} \\
& \times\left(1+O_{a, k}\left(\frac{1}{d_{1}}+\frac{1}{d_{2}}+\frac{d_{1} d_{2}}{\left(d_{1}+d_{2}\right)^{2}}\right)\right) .
\end{aligned}
$$

Note that the last formula in Theorem 2.4 does not give asymptotic behavior if $d_{1}$ and $d_{2}$ grow so that $d_{2} / d_{1}$ tends to a finite nonzero limit. The precise bounds show that the term $\left(d_{1}+d_{2}\right)^{1-a} / d_{1}^{2} d_{2}^{2}$ still gives the correct order of growth for $c_{X}$, but the coefficient can differ from

$$
k a(a+1) \frac{\Gamma(k a+a+1)}{\Gamma(k a)} .
$$

And in fact, the coefficient differs.

Theorem 2.5. Let $d_{1}, d_{2} \geq k, c>0$. Then

$$
P\left(\left|X\left(d_{1}, d_{2}, t\right)-E X\left(d_{1}, d_{2}, t\right)\right| \geq c\left(d_{1}+d_{2}\right) \sqrt{k t}\right) \leq 2 \exp \left(-\frac{c^{2}}{8}\right) .
$$

In particular, if $c(t) \rightarrow \infty$ as $t \rightarrow \infty$, then $|X-E X|<c(t)\left(d_{1}+d_{2}\right) \sqrt{k t}$ with probability tending to 1 .

From Theorems 2.4 and 2.5, we immediately obtain the following assertion.

Corollary 2.6. If $\left(d_{1}+d_{2}\right)^{a} d_{1}^{2} d_{2}^{2}=o(\sqrt{t})$, then with probability tending to 1 as $d_{1}, d_{2}, t \rightarrow \infty$,

$$
X\left(d_{1}, d_{2}, t\right) \sim c_{X}\left(d_{1}, d_{2}\right) t
$$


The mean value of the number of edges between one node with degree $d_{1}$ and another node with degree $d_{2}$ is $X\left(d_{1}, d_{2}, t\right) / R\left(d_{1}, t\right) R\left(d_{2}, t\right)$. Since the quantities $R(d, t)$ and $X\left(d_{1}, d_{2}, t\right)$ are tightly concentrated around their expectations, the main term of the ratio is

$$
\frac{\Gamma(k a+1)}{(a+1) \Gamma(k a+a+1)} \frac{d_{1}^{a} d_{2}^{a}\left(d_{1}+d_{2}\right)^{1-a}}{t} .
$$

Again, the constant factor can differ if $d_{1}$ and $d_{2}$ grow so that $d_{2} / d_{1}$ tends to a finite nonzero limit, but the order is correct even in this case.

\section{Proof of Theorem 2.1}

For a property $P$, we define

$$
[P]= \begin{cases}1, & P \text { holds } \\ 0, & \text { otherwise }\end{cases}
$$

First of all, we reformulate the model without reference to $H_{a, 1}^{(t)}$. The probability space $H_{a, k}^{(1)}$ obviously consists of one graph with one node and $k$ loops. The space $H_{a, k}^{(t+1)}$ can be obtained from $H_{a, k}^{(t)}$ by adding to any graph from $H_{a, k}^{(t)}$ a new node $t+1$ and $k$ edges in the following $k$ steps. At the $i$ th step, we add one edge between the new node and one of the existing nodes $\gamma$. If $\gamma \neq t+1$, then it corresponds to a group of nodes $\gamma_{1}, \ldots, \gamma_{k}$ in $H_{a, 1}^{(k t+i-1)}$. The sum of degrees of $\gamma_{1}, \ldots, \gamma_{k}$ equals the degree of $\gamma$ in the graph before the $i$ th step. We denote this degree by $\operatorname{deg}_{t, i}$. So

$$
\operatorname{Pr}(\gamma=s)=\frac{\operatorname{deg}_{t, i}(s)+k(a-1)}{(a+1)(k t+i)-1}, \quad 1 \leq s \leq t .
$$

If $\gamma=t+1$, the corresponding group in $H_{a, 1}^{(k t+i-1)}$ has only $i-1$ nodes. Hence,

$$
\operatorname{Pr}(\gamma=t+1)=\frac{\operatorname{deg}_{t, i}(t+1)+(i-1)(a-1)+a}{(a+1)(k t+i)-1} .
$$

We want to express any value $r(d, t)$ in terms of some values with smaller $t$. Let us consider the transition from $H_{a, k}^{(t)}$ to $H_{a, k}^{(t+1)}$. Let $r(d, t, i)$ denote the average number of nodes of degree $d$, not including the last node $t+1$ before the $i$ th step, and $r(d, t, i+1)$, the similar number after the $i$ th step. Let $\gamma$ be a head of 
the edge added in the $i$ th step. Then

$$
\begin{aligned}
r(d, t, i+1)= & \sum_{s=1}^{t} \operatorname{Pr}\left(\operatorname{deg}_{t, i+1}(s)=d\right) \\
= & \sum_{s=1}^{t}\left(\operatorname{Pr}\left(\operatorname{deg}_{t, i+1}(s)=d, \gamma=s\right)+\operatorname{Pr}\left(\operatorname{deg}_{t, i+1}(s)=d, \gamma \neq s\right)\right) \\
= & \sum_{s=1}^{t}\left(\operatorname{Pr}\left(\operatorname{deg}_{t, i}(s)=d-1, \gamma=s\right)+\operatorname{Pr}\left(\operatorname{deg}_{t, i}(s)=d, \gamma \neq s\right)\right) \\
= & \sum_{s=1}^{t}\left(\operatorname{Pr}\left(\operatorname{deg}_{t, i}(s)=d-1\right) \frac{(d-1)+k(a-1)}{(a+1)(k t+i)-1}\right. \\
= & \quad+\operatorname{Pr}(d-1, t, i) \frac{(d-1)+k(a-1)}{(a+1)(k t+i)-1} \\
& \left.+r(d, t, i)\left(1-\frac{d+k(a-1)(k t+i)-1}{(a+1)(k t+i)-1}\right)\right)
\end{aligned}
$$

By definition,

$$
r(d, t)=r(d, t, 1), \quad r(d, t+1)=r(d, t, k+1)+\operatorname{Pr}\left(\operatorname{deg}_{t, k+1}(t+1)=d\right) .
$$

The function $r(d, t)$ is completely determined by equations (3.1), (3.2) and the starting condition

$$
r(d, 1)=[d=2 k] .
$$

Equation (3.2) includes the function $\operatorname{Pr}\left(\operatorname{deg}_{t, k+1}(t+1)=d\right)$. Obviously,

$$
\operatorname{Pr}\left(\operatorname{deg}_{t, k+1}(t+1)=d\right)=0, \quad d<k \text { or } d>2 k .
$$

The minimal value $\operatorname{deg}_{t, k+1}(t+1)=k$ is obtained when none of the $k$ edges is a loop. In this case, $\operatorname{deg}_{t, i}(t+1)=i-1$ for all $i$, so

$$
\operatorname{Pr}\left(\operatorname{deg}_{t, k+1}(t+1)=k\right)=\prod_{i=1}^{k}\left(1-\frac{i a}{(a+1)(k t+i)-1}\right)=1+O\left(\frac{1}{t}\right) .
$$

(Note that the constant in $O()$ depends on $a$ and $k$.)

Because $\sum_{d=k}^{2 k} \operatorname{Pr}\left(\operatorname{deg}_{t, k+1}=d\right)=1$ and $\operatorname{Pr}\left(\operatorname{deg}_{t, k+1}=d\right) \geq 0$, we get

$$
\operatorname{Pr}\left(\operatorname{deg}_{t, k+1}(t+1)=d\right)=O\left(\frac{1}{t}\right), \quad k<d \leq 2 k .
$$

Since $d$ is bounded in the last equality, its right-hand side can be equivalently written as $O\left(1 / d^{2} t\right)$. 
Let

$$
c(d)= \begin{cases}\frac{\mathrm{B}(d-k+k a, a+2)}{\mathrm{B}(k a, a+1)}, & d \geq k, \\ 0, & d<k .\end{cases}
$$

If $d>k$, then

$$
\begin{aligned}
\frac{c(d-1)}{c(d)} & =\frac{\mathrm{B}(d-1-k+k a, a+2)}{\mathrm{B}(d-k+k a, a+2)} \\
& =\frac{\Gamma(d-1-k+k a) / \Gamma(d+1-k+k a+a)}{\Gamma(d-k+k a) / \Gamma(d-k+k a+a+2)} \\
& =\frac{d+1-k+k a+a}{d-1-k+k a} .
\end{aligned}
$$

Also

$$
c(k)=\frac{\mathrm{B}(k a, a+2)}{\mathrm{B}(k a, a+1)}=\frac{\Gamma(a+2) / \Gamma(k a+a+2)}{\Gamma(a+1) / \Gamma(k a+a+1)}=\frac{a+1}{k a+a+1} .
$$

In particular, $c(d-1)>c(d)$, so $c(d)<c(k)<1$ for all $d \geq k$.

For the rest of the proof, we will assume that $d \geq k$. Note that of course this does not imply $d-1 \geq k$.

As $d$ grows, the asymptotic behavior of $c(d)$ is

$$
\begin{aligned}
\ln c(d)= & \ln \frac{\Gamma(a+2)}{\mathrm{B}(k a, a+1)} \frac{\Gamma(d-k+k a)}{\Gamma(d-k+k a+a+2)} \\
= & \ln \frac{\Gamma(a+2)}{\mathrm{B}(k a, a+1)}+(d-k+k a)(\ln (d-k+k a)-1) \\
& \quad-(d-k+k a+a+2)(\ln (d-k+k a+a+2)-1)+O\left(\frac{1}{d}\right) \\
= & \ln \frac{\Gamma(a+2)}{\mathrm{B}(k a, a+1)}+(d-k+k a)\left(\ln d+\frac{-k+k a}{d}-1\right) \\
& \quad-(d-k+k a+a+2)\left(\ln d+\frac{-k+k a+a+2}{d}-1\right)+O\left(\frac{1}{d}\right) \\
= & \ln \frac{\Gamma(a+2)}{\mathrm{B}(k a, a+1)}-(a+2) \ln d+O\left(\frac{1}{d}\right),
\end{aligned}
$$

and

$$
c(d)=\frac{\Gamma(a+2)}{\mathrm{B}(k a, a+1)} d^{-2-a}\left(1+O\left(\frac{1}{d}\right)\right) .
$$

Let

$$
\tilde{r}(d, t, i)=r(d, t, i)-c(d)\left(t+\frac{i}{k}-\frac{1}{k(a+1)}\right) .
$$


It is easy to see that the theorem is equivalent to $\tilde{r}(d, t, i)=O(1)$. Using (3.1), we obtain

$$
\begin{aligned}
\tilde{r}(d-1, t, i) & (d-1)+k(a-1) \\
(a+1)(k t+i)-1 & \tilde{r}(d, t, i)\left(1-\frac{d+k(a-1)}{(a+1)(k t+i)-1}\right) \\
= & r(d, t, i+1)-\left(t+\frac{i}{k}-\frac{1}{k(a+1)}\right) \\
& \quad \times\left(c(d-1) \frac{(d-1)+k(a-1)}{(a+1)(k t+i)-1}+c(d)\left(1-\frac{d+k(a-1)}{(a+1)(k t+i)-1)}\right)\right) \\
= & r(d, t, i+1)-\left(t+\frac{i}{k}-\frac{1}{k(a+1)}\right) c(d) \\
& \times \frac{(1-[d=k])(d+1-k+k a+a)+(a+1)(k t+i)-1-(d+k(a-1))}{(a+1)(k t+i)-1} \\
= & r(d, t, i+1)-c(d) \frac{(a+1)(k t+i+1)-1-[d=k](1+k a+a)}{k(a+1)} \\
= & \tilde{r}(d, t, i+1)+[d=k] c(k) \frac{1+k a+a}{k(a+1)}=\tilde{r}(d, t, i+1)+\frac{[d=k]}{k} .
\end{aligned}
$$

Let $C=C(a, k)$ be a sufficiently large constant that will be determined later. We claim that

$$
\left|\tilde{r}(d, t, i)+(i-1) \frac{[d=k]}{k}\right| \leq \frac{C}{d+k a}\left(1-\frac{\min \{1, k a\}}{(a+2)(t+1)(d+k a)}\right)^{i-1}
$$

for all $i=1, \ldots, k+1$ and for all natural numbers $d \geq k$ and $t$. Note that this implies $\tilde{r}(d, t, i)=O(1 / d)$ and Theorem 2.1.

Equations (3.1), (3.2), (3.3), (3.4) imply that $r(d, t, i)=0$ if $d>k t+i-1+k$. In this case, using (3.6), we obtain

$$
\tilde{r}(d, t, i)=-c(d)\left(t+\frac{i}{k}-\frac{1}{k(a+1)}\right)=O\left(d^{-2-a} t\right)=O\left(d^{-1-a}\right),
$$

so if $d>k t+i-1+k,(3.8)$ holds for all sufficiently large values of $C$.

Now assume $d \leq k t+i-1+k$. We will prove (3.8) by induction on $t$ and, for fixed $t$, on $i$. The basis of induction $t=1, \ldots, 1+\lfloor 1 / k a\rfloor$ and any $i=1, \ldots, k+1$ obviously holds for all sufficiently large values of $C$.

Now let $t \geq 2+\lfloor 1 / k a\rfloor$ and let (3.8) hold for $t-1$. Using (3.2), we obtain

$$
\begin{gathered}
\tilde{r}(d, t, 1)=\tilde{r}(d, t-1, k+1)+\operatorname{Pr}\left(\operatorname{deg}_{t-1, k+1}(t)=d\right) \\
=\tilde{r}(d, t-1, k+1)+[d=k]+O\left(\frac{1}{d^{2} t}\right) .
\end{gathered}
$$


Therefore,

$$
\begin{aligned}
|\tilde{r}(d, t, 1)| & \leq \frac{C}{d+k a}\left(1-\frac{\min \{1, k a\}}{(a+2)(t+1)(d+k a)}\right)^{k}+O\left(\frac{1}{d^{2} t}\right) \\
& \leq \frac{C}{d+k a}-\frac{C \min \{1, k a\} /(a+2)}{(t+1)(d+k a)^{2}}+O\left(\frac{1}{d^{2} t}\right) .
\end{aligned}
$$

Thus the induction step on $t$ is proved for all sufficiently large values of $C$.

Finally, let $t \geq 2+\lfloor 1 / k a\rfloor>1+1 / k a, i>1$, and let (3.8) hold for $i-1$. We temporarily set $T=(a+1)(k t+i-1)-1$. Note that $T$ depends on $t$ and $i$, but not on $d$. From (3.7) we obtain

$$
\begin{aligned}
\tilde{r}(d, t, i) & +(i-1) \frac{[d=k]}{k} \\
= & (i-2) \frac{[d=k]}{k}+\tilde{r}(d-1, t, i-1) \frac{(d-1)+k(a-1)}{T} \\
& +\tilde{r}(d, t, i-1)\left(1-\frac{d+k(a-1)}{T}\right) \\
= & \left(\tilde{r}(d-1, t, i-1)+(i-2) \frac{[d-1=k]}{k}\right) \frac{(d-1)+k(a-1)}{T} \\
& +\left(\tilde{r}(d, t, i-1)+(i-2) \frac{[d=k]}{k}\right)\left(1-\frac{d+k(a-1)}{T}\right) \\
& +[k \leq d \leq k+1] O\left(\frac{1}{t}\right) .
\end{aligned}
$$

The remainder term $[k \leq d \leq k+1] O(1 / t)$ can be written as $O\left(1 / d^{2} t\right)$. The assumptions $d \leq k t+i-1+k$ and $t>1+1 / k a$ imply that

$$
1-\frac{d+k(a-1)}{(a+1)(k t+i-1)-1} \geq 0 .
$$

If $d>k$, then

$$
\begin{aligned}
& \frac{C}{d-1+k a} \frac{(d-1)+k(a-1)}{T}+\frac{C}{d+k a}\left(1-\frac{d+k(a-1)}{T}\right) \\
= & \frac{C}{d+k a}\left(1-\frac{1}{T}\left(-\frac{d+k a}{d-1+k a}((d-1)+k(a-1))+d+k(a-1)\right)\right) \\
= & \frac{C}{d+k a}\left(1-\frac{1}{T}\left(1-\frac{(d-1)+k(a-1)}{d-1+k a}\right)\right) \\
= & \frac{C}{d+k a}\left(1-\frac{k}{T(d-1+k a)}\right) \leq \frac{C}{d+k a}\left(1-\frac{k \min \{1, k a\}}{T(d+k a)}\right) .
\end{aligned}
$$


If $d=k$, then

$$
\begin{aligned}
\frac{C}{d+k a}\left(1-\frac{d+k(a-1)}{T}\right) & =\frac{C}{d+k a}\left(1-\frac{k a(k+k a)}{T(d+k a)}\right) \\
& \leq \frac{C}{d+k a}\left(1-\frac{k \min \{1, k a\}}{T(d+k a)}\right) .
\end{aligned}
$$

In both cases,

$$
\begin{aligned}
& \left|\tilde{r}(d, t, i)+(i-1) \frac{[d=k]}{k}\right| \\
& \quad \leq \frac{C}{d+k a}\left(1-\frac{\min \{1, k a\}}{(a+2)(t+1)(d+k a)}\right)^{i-2}\left(1-\frac{k \min \{1, k a\}}{T(d+k a)}\right)+O\left(\frac{1}{d^{2} t}\right) .
\end{aligned}
$$

Note that

$$
\begin{aligned}
1 & -\frac{k \min \{1, k a\}}{((a+1)(k t+i-1)-1)(d+k a)} \\
& =\left(1-\frac{k \min \{1, k a\}}{(a+2)(k t+k)(d+k a)}\right)-\frac{k \min \{1, k a\}}{(a+1)(a+2) k t(d+k a)}\left(1+O\left(\frac{1}{t}\right)\right),
\end{aligned}
$$

where the left-hand side is strictly less than the first term on the right-hand side, so that the second term on the right-hand side is positive. Now (3.9) implies (3.8) for all sufficiently large values of $C$.

Theorem 2.1 is proved.

\section{Proof of Theorem 2.2}

By definition and linearity of expectation,

$$
\begin{aligned}
\operatorname{cov} & \left(R\left(d_{1}, t\right), R\left(d_{2}, t\right)\right) \\
& =E\left(R\left(d_{1}, t\right) R\left(d_{2}, t\right)\right)-r\left(d_{1}, t\right) r\left(d_{2}, t\right) \\
& =E \sum_{s_{1}, s_{2}=1}^{t}\left[\operatorname{deg} s_{1}=d_{1}, \operatorname{deg} s_{2}=d_{2}\right]-r\left(d_{1}, t\right) r\left(d_{2}, t\right) \\
& =\sum_{s_{1} \neq s_{2}} \operatorname{Pr}\left(\operatorname{deg} s_{1}=d_{1}, \operatorname{deg} s_{2}=d_{2}\right)+\left[d_{1}=d_{2}\right] r\left(d_{1}, t\right)-r\left(d_{1}, t\right) r\left(d_{2}, t\right) .
\end{aligned}
$$

We will estimate the sum

$$
r_{2}\left(d_{1}, d_{2}, t\right)=\sum_{\substack{s_{1}, s_{2}=1 \\ s_{1} \neq s_{2}}}^{t} \operatorname{Pr}\left(\operatorname{deg} s_{1}=d_{1}, \operatorname{deg} s_{2}=d_{2}\right)
$$

as we did for the function $r(d, t)$ in the proof of Theorem 2.1. 
As with $r(d, t)$, we define a function $r_{2}\left(d_{1}, d_{2}, t, i\right)$ as the value of $r_{2}\left(d_{1}, d_{2}, t\right)$ before the $i$ th step in the transition from $H_{a, k}^{(t)}$ to $H_{a, k}^{(t+1)}$. The recurrent equation is deduced similarly to (3.1). For fixed $s_{1}$ and $s_{2}$, there are three nonintersecting cases: $\gamma=s_{1}, \gamma=s_{2}$, and $\gamma \notin\left\{s_{1}, s_{2}\right\}$. In the first case, we get

$$
\begin{gathered}
\operatorname{Pr}\left(\operatorname{deg}_{t, i+1}\left(s_{1}\right)=d_{1}, \operatorname{deg}_{t, i+1}\left(s_{2}\right)=d_{2}, \gamma=s_{1}\right) \\
=\operatorname{Pr}\left(\operatorname{deg}_{t, i}\left(s_{1}\right)=d_{1}-1, \operatorname{deg}_{t, i+1}\left(s_{2}\right)=d_{2}, \gamma=s_{1}\right) \\
=\operatorname{Pr}\left(\operatorname{deg}_{t, i}\left(s_{1}\right)=d_{1}-1, \operatorname{deg}_{t, i+1}\left(s_{2}\right)=d_{2}\right) \frac{d_{1}-1+k(a-1)}{(a+1)(k t+i)-1}
\end{gathered}
$$

The second case is the same with $d_{1}$ and $d_{2}$ interchanged. In the third case, we get

$$
\begin{aligned}
& \operatorname{Pr}\left(\operatorname{deg}_{t, i+1}\left(s_{1}\right)=d_{1}, \operatorname{deg}_{t, i+1}\left(s_{2}\right)=d_{2}, \gamma \neq s_{1}, \gamma \neq s_{2}\right) \\
& \quad=\operatorname{Pr}\left(\operatorname{deg}_{t, i}\left(s_{1}\right)=d_{1}, \operatorname{deg}_{t, i+1}\left(s_{2}\right)=d_{2}, \gamma \neq s_{1}, \gamma \neq s_{2}\right) \\
& \quad=\operatorname{Pr}\left(\operatorname{deg}_{t, i}\left(s_{1}\right)=d_{1}, \operatorname{deg}_{t, i+1}\left(s_{2}\right)=d_{2}\right)\left(1-\frac{d_{1}+k(a-1)+d_{2}+k(a-1)}{(a+1)(k t+i)-1}\right)
\end{aligned}
$$

so the final formula is

$$
\begin{aligned}
r_{2}\left(d_{1}, d_{2}, t, i+1\right)=r_{2} & \left(d_{1}-1, d_{2}, t, i\right) \frac{\left(d_{1}-1\right)+k(a-1)}{(a+1)(k t+i)-1} \\
& +r_{2}\left(d_{1}, d_{2}-1, t, i\right) \frac{\left(d_{2}-1\right)+k(a-1)}{(a+1)(k t+i)-1} \\
& +r_{2}\left(d_{1}, d_{2}, t, i\right)\left(1-\frac{d_{1}+d_{2}+2 k(a-1)}{(a+1)(k t+i)-1}\right) .
\end{aligned}
$$

By definition,

$$
\begin{aligned}
r_{2}\left(d_{1}, d_{2}, t\right)= & r_{2}\left(d_{1}, d_{2}, t, 1\right), \\
r_{2}\left(d_{1}, d_{2}, t+1\right)= & r_{2}\left(d_{1}, d_{2}, t, k+1\right) \\
& +\sum_{s=1}^{t} \operatorname{Pr}\left(\operatorname{deg}_{t, k+1}(s)=d_{1}, \operatorname{deg}_{t, k+1}(t+1)=d_{2}\right) \\
& +\sum_{s=1}^{t} \operatorname{Pr}\left(\operatorname{deg}_{t, k+1}(s)=d_{2}, \operatorname{deg}_{t, k+1}(t+1)=d_{1}\right),
\end{aligned}
$$

and the starting condition is

$$
r_{2}\left(d_{1}, d_{2}, 1\right)=0 .
$$

Equation (4.3) includes a function

$$
r_{2}^{\prime}\left(d_{1}, d_{2}, t, i\right)=\sum_{s=1}^{t} \operatorname{Pr}\left(\operatorname{deg}_{t, i}(s)=d_{1}, \operatorname{deg}_{t, i}(t)=d_{2}\right)
$$


(and the same function with swapped arguments), so we will first estimate $r_{2}^{\prime}$. Again, we write a recurrent equation. For fixed $s$, there are three nonintersecting cases: $\gamma=s, \gamma=t+1, \gamma \notin\{s, t+1\}$. If $\operatorname{deg}_{t, i+1}(s)=d_{1}$, then $\operatorname{deg}_{t, i}(s)$ equals $d_{1}-1$ in the first case and $d_{1}$ in the other two cases. If $\operatorname{deg}_{t, i+1}(t+1)=d_{2}$, then $\operatorname{deg}_{t, i}(t+1)=d_{2}-2$ in the second case and $d_{2}-1$ in the other two cases. Calculating probabilities, we obtain

$$
\begin{aligned}
& r_{2}^{\prime}\left(d_{1}, d_{2}, t, i+1\right) \\
&=r_{2}^{\prime}\left(d_{1}-1, d_{2}-1, t, i\right) \frac{\left(d_{1}-1\right)+k(a-1)}{(a+1)(k t+i)-1} \\
&+r_{2}^{\prime}\left(d_{1}, d_{2}-2, t, i\right) \frac{\left(d_{2}-2\right)+(i-1)(a-1)+a}{(a+1)(k t+i)-1} \\
&+r_{2}^{\prime}\left(d_{1}, d_{2}-1, t, i\right)\left(1-\frac{d_{1}+k(a-1)+\left(d_{2}-1\right)+(i-1)(a-1)+a}{(a+1)(k t+i)-1}\right) .
\end{aligned}
$$

Before the first step, the node $t$ has degree 0 , so

$$
r_{2}^{\prime}\left(d_{1}, d_{2}, t, 1\right)=\sum_{s=1}^{t} \operatorname{Pr}\left(\operatorname{deg}_{t, 1}(s)=d_{1}\right)\left[d_{2}=0\right]=\left[d_{2}=0\right] r\left(d_{1}, t\right) .
$$

We continue to use the notation (3.5). Obviously, $r_{2}^{\prime}\left(d_{1}, d_{2}, t, i\right)=0$ when $d_{2}>$ $2(i-1)$ or $d_{1} \geq 2(k t+i)$. If $d_{1}<2(k t+i)$ and $d_{2} \leq 2(i-1)$, then

$$
\frac{d_{1}+d_{2}}{t} \cdot O\left(d_{1}^{-a-2} t\right)=O\left(d_{1}^{-a-1}\right)=O\left(d_{1}^{-1}\right)
$$

and

$$
\frac{d_{1}+d_{2}}{t} \cdot O\left(d_{1}^{-1}\right)=O\left(d_{1}^{-1}\right)
$$

Now it is easy to see that

$$
r_{2}^{\prime}\left(d_{1}, d_{2}, t, i\right)=\left[d_{2}=i-1\right] c\left(d_{1}\right) t+O\left(d_{1}^{-1}\right) .
$$

Since $r_{2}^{\prime}\left(d_{1}, d_{2}, t, i\right)=0$ when $d_{2} \geq 2 k$, the remainder term in (4.5) is zero when $d_{2} \geq 2 k$ and can be written as $O\left(1 / d_{1} d_{2}^{a+1}\right)$.

Since $r\left(d_{1}, t\right)=0$ when $d_{1}>k t+k, r_{2}^{\prime}\left(d_{1}, d_{2}, t, i\right)=0$ when $d_{1}+d_{2}>k t+$ $k+2(i-1)$. In particular, $r_{2}^{\prime}\left(d_{1}, d_{2}, t, k+1\right)+r_{2}^{\prime}\left(d_{2}, d_{1}, t, k+1\right)=0$ when $d_{1}+$ $d_{2}>k t+2 k$. By (4.2) and (4.3), $r_{2}\left(d_{1}, d_{2}, t, i\right)=0$ when $d_{1}+d_{2}>k t+2 k+$ $(i-1)$.

For the rest of the proof, we will assume that $d_{1} \geq k$ and $d_{2} \geq k$. Note that of course this does not imply $d_{1}-1 \geq k$ and $d_{2}-1 \geq k$. 
Let

$$
\begin{aligned}
& \tilde{r}_{2}\left(d_{1}, d_{2}, t, i\right) \\
& \quad=r_{2}\left(d_{1}, d_{2}, t, i\right)-c\left(d_{1}\right) c\left(d_{2}\right)\left(t+\frac{i}{k}-\frac{1}{k(a+1)}\right)\left(t+\frac{i+1}{k}-\frac{1}{k(a+1)}\right) .
\end{aligned}
$$

We temporarily set $T=k t+i-1 /(a+1)$. We express $r_{2}$ in terms of $\tilde{r}_{2}$ and use (4.2). In the expression, there are terms with $\tilde{r}_{2}$ with various arguments. Now we transform the terms without $\tilde{r}_{2}$ from the right-hand side of (4.2):

$$
\begin{gathered}
\frac{T}{k} \frac{T+1}{k}\left(c\left(d_{1}-1\right) c\left(d_{2}\right) \frac{\left(d_{1}-1\right)+k(a-1)}{(a+1) T}+c\left(d_{1}\right) c\left(d_{2}-1\right) \frac{\left(d_{2}-1\right)+k(a-1)}{(a+1) T}\right. \\
\left.\quad+c\left(d_{1}\right) c\left(d_{2}\right)\left(1-\frac{d_{1}+d_{2}+2 k(a-1)}{(a+1) T}\right)\right) \\
=\frac{T}{k} \frac{T+1}{k} c\left(d_{1}\right) c\left(d_{2}\right)\left(\left(1-\left[d_{1}=k\right]\right) \frac{d_{1}+1-k+k a+a}{(a+1) T}\right. \\
\left.\quad+\left(1-\left[d_{2}=k\right]\right) \frac{d_{2}+1-k+k a+a}{(a+1) T}+1-\frac{d_{1}+d_{2}+2 k(a-1)}{(a+1) T}\right) \\
=\frac{T}{k} \frac{T+1}{k} c\left(d_{1}\right) c\left(d_{2}\right) \frac{1}{(a+1) T}\left(2(a+1)+(a+1) T-\left[d_{1}=k\right](1+k a+a)\right. \\
=\frac{T+1}{k} \frac{T+2}{k} c\left(d_{1}\right) c\left(d_{2}\right)-\left[d_{1}=k\right] \frac{(T+1) c\left(d_{2}\right)}{k^{2}}-\left[d_{2}=k\right] \frac{(T+1) c\left(d_{1}\right)}{k^{2}} .
\end{gathered}
$$

The first term equals the term without $\tilde{r}_{2}$ on the left-hand side, so

$$
\begin{gathered}
\tilde{r}_{2}\left(d_{1}, d_{2}, t, i+1\right)=\tilde{r}_{2}\left(d_{1}-1, d_{2}, t, i\right) \frac{\left(d_{1}-1\right)+k(a-1)}{(a+1)(k t+i)-1} \\
+\tilde{r}_{2}\left(d_{1}, d_{2}-1, t, i\right) \frac{\left(d_{2}-1\right)+k(a-1)}{(a+1)(k t+i)-1} \\
+\tilde{r}_{2}\left(d_{1}, d_{2}, t, i\right)\left(1-\frac{d_{1}+d_{2}+2 k(a-1)}{(a+1)(k t+i)-1}\right) \\
-\left[d_{1}=k\right] \frac{\left(k t+i-\frac{1}{a+1}+1\right) c\left(d_{2}\right)}{k^{2}} \\
-\left[d_{2}=k\right] \frac{\left(k t+i-\frac{1}{a+1}+1\right) c\left(d_{1}\right)}{k^{2}} .
\end{gathered}
$$


Relations (4.3) and (4.5) imply

$$
\begin{gathered}
\tilde{r}_{2}\left(d_{1}, d_{2}, t, 1\right)=\tilde{r}_{2}\left(d_{1}, d_{2}, t-1, k+1\right)+\left[d_{2}=k\right] c\left(d_{1}\right) t+\left[d_{1}=k\right] c\left(d_{2}\right) t \\
+O\left(\frac{1}{d_{1}^{a+1} d_{2}}+\frac{1}{d_{1} d_{2}^{a+1}}\right)
\end{gathered}
$$

Let

$$
c_{1}\left(d_{1}^{\prime}, d_{2}^{\prime}\right)= \begin{cases}\frac{\Gamma\left(d_{1}^{\prime}-k+k a\right)}{\left(d_{2}^{\prime}+k(a-1)\right) \Gamma\left(d_{1}^{\prime}-k+k a+a+1\right)}, & d_{1}^{\prime} \geq k, d_{2}^{\prime} \geq k, \\ 0, & d_{1}^{\prime}<k \text { or } d_{2}^{\prime}<k .\end{cases}
$$

By definition, for $d_{1}>k$,

$$
\frac{c_{1}\left(d_{1}-1, d_{2}\right)}{c_{1}\left(d_{1}, d_{2}\right)}=\frac{d_{1}-k+k a+a}{d_{1}-k+k a-1} .
$$

For $d_{2}>k$,

$$
\frac{c_{1}\left(d_{1}, d_{2}-1\right)}{c_{1}\left(d_{1}, d_{2}\right)}=\frac{d_{2}+k(a-1)}{d_{2}-1+k(a-1)} .
$$

Similar to (3.6), we have

$$
c_{1}\left(d_{1}, d_{2}\right)=\frac{d_{1}^{-1-a}}{d_{2}+k(a-1)}\left(1+O\left(\frac{1}{d_{1}}\right)\right) .
$$

Moreover,

$$
\begin{aligned}
\frac{c_{1}\left(d_{1}, d_{2}\right)}{c\left(d_{1}\right)} & =\frac{\mathrm{B}(k a, a+1)}{\Gamma(a+2)} \frac{\Gamma\left(d_{1}-k+k a\right) \Gamma\left(d_{1}-k+k a+a+2\right)}{\left(d_{2}+k(a-1)\right) \Gamma\left(d_{1}-k+k a+a+1\right) \Gamma\left(d_{1}-k+k a\right)} \\
& =\frac{\mathrm{B}(k a, a+1)}{\Gamma(a+2)} \frac{d_{1}-k+k a+a+1}{d_{2}+k(a-1)} .
\end{aligned}
$$

Let $C=C(a, k)$ be a sufficiently large constant that will be determined later. We claim that

$$
\begin{aligned}
& \left|\tilde{r}_{2}\left(d_{1}, d_{2}, t, i\right)+\frac{i-1}{k}\left(\left[d_{2}=k\right] c\left(d_{1}\right)+\left[d_{1}=k\right] c\left(d_{2}\right)\right) t\right| \\
& \quad \leq C\left(c_{1}\left(d_{1}, d_{2}\right)+c_{1}\left(d_{2}, d_{1}\right)\right)\left(k t+\frac{a+\frac{1}{2}}{a+1} i\right)
\end{aligned}
$$

for all $i=1, \ldots, k+1$ and for all natural numbers $d_{1} \geq k, d_{2} \geq k, t$. Since both sides of (4.6) are symmetric in $d_{1}$ and $d_{2}$, it is sufficient to consider the case $d_{1} \leq d_{2}$. 
If $d_{1}+d_{2}>k t+2 k+(i-1)$, then $r_{2}\left(d_{1}, d_{2}, t, i\right)=0$ and

$$
\begin{aligned}
\frac{\left|\tilde{r}_{2}\left(d_{1}, d_{2}, t, i\right)\right|}{c_{1}\left(d_{1}, d_{2}\right) t} & =\frac{c\left(d_{1}\right) c\left(d_{2}\right)\left(t+\frac{i}{k}-\frac{1}{k(a+1)}\right)\left(t+\frac{i+1}{k}-\frac{1}{k(a+1)}\right)}{c_{1}\left(d_{1}, d_{2}\right) t} \\
& =O\left(\frac{d_{2}+k(a-1)}{d_{1}-k+k a+a+1} c\left(d_{2}\right) t\right)=O\left(\frac{t}{d_{1} d_{2}^{1+a}}\right) .
\end{aligned}
$$

Since

$$
d_{2} \geq \frac{d_{1}+d_{2}}{2} \geq \frac{k}{2} t+k
$$

the right-hand side is bounded. Obviously, $\left[d_{2}=k\right]=0$, and

$$
\left[d_{1}=k\right] c\left(d_{2}\right) t=O\left(\frac{t}{d_{2}^{2+a}}\right)
$$

is bounded too. Thus (4.6) holds when $d_{1}+d_{2}>k t+2 k+(i-1)$ for all sufficiently large values of $C$.

Let $d_{1}+d_{2} \leq k t+2 k+(i-1)$. We will prove (4.6) by induction on $t$, and for fixed $t$, on $i$. The basis of induction $t=1, \ldots, 2+\lfloor 1 / k a\rfloor$ and any $i=1, \ldots, k+1$ obviously holds for all sufficiently large values of $C$.

Let $t \geq 3+\lfloor 1 / k a\rfloor$ and let (4.6) hold for $t-1$. We continue to use the restriction $d_{1} \leq d_{2}$. Thus,

$$
\begin{aligned}
\left|\tilde{r}_{2}\left(d_{1}, d_{2}, t, 1\right)\right|=\mid & \tilde{r}_{2}\left(d_{1}, d_{2}, t-1, k+1\right)+\left[d_{2}=k\right] c\left(d_{1}\right) t+\left[d_{1}=k\right] c\left(d_{2}\right) t \\
& +O\left(\frac{1}{d_{1}^{a+1} d_{2}}\right) \mid \\
\leq & C\left(c_{1}\left(d_{1}, d_{2}\right)+c_{1}\left(d_{2}, d_{1}\right)\right)\left(k(t-1)+\frac{a+\frac{1}{2}}{a+1}(k+1)\right) \\
& +O\left(\frac{1}{d_{1}^{a+1} d_{2}}\right) .
\end{aligned}
$$

Since $c_{1}\left(d_{1}, d_{2}\right)=O\left(1 / d_{1}^{a+1} d_{2}\right)$, the right-hand side is less than

$$
C\left(c_{1}\left(d_{1}, d_{2}\right)+c_{1}\left(d_{2}, d_{1}\right)\right)\left(k t+\frac{a+\frac{1}{2}}{a+1}\right)
$$

for all sufficiently large values of $C$. This completes the induction on $t$. 
Let $t \geq 3+\lfloor 1 / k a\rfloor>2+1 / k a, i>1$, and let (4.6) hold for $i-1$. Then

$$
\begin{aligned}
& \tilde{r}_{2}\left(d_{1}, d_{2}, t, i\right)+\frac{i-1}{k}\left(\left[d_{2}=k\right] c\left(d_{1}\right)+\left[d_{1}=k\right] c\left(d_{2}\right)\right) t \\
& =\tilde{r}_{2}\left(d_{1}-1, d_{2}, t, i-1\right) \frac{\left(d_{1}-1\right)+k(a-1)}{(a+1)(k t+i-1)-1} \\
& +\tilde{r}_{2}\left(d_{1}, d_{2}-1, t, i-1\right) \frac{\left(d_{2}-1\right)+k(a-1)}{(a+1)(k t+i-1)-1} \\
& +\tilde{r}_{2}\left(d_{1}, d_{2}, t, i-1\right)\left(1-\frac{d_{1}+d_{2}+2 k(a-1)}{(a+1)(k t+i-1)-1}\right) \\
& -\left[d_{1}=k\right] \frac{\left(k t+i-1-\frac{1}{a+1}+1\right) c\left(d_{2}\right)}{k^{2}} \\
& -\left[d_{2}=k\right] \frac{\left(k t+i-1-\frac{1}{a+1}+1\right) c\left(d_{1}\right)}{k^{2}} \\
& +\frac{i-1}{k}\left(\left[d_{2}=k\right] c\left(d_{1}\right)+\left[d_{1}=k\right] c\left(d_{2}\right)\right) t \\
& =\left(\tilde{r}_{2}\left(d_{1}-1, d_{2}, t, i-1\right)+\frac{i-2}{k}\left(\left[d_{2}=k\right] c\left(d_{1}-1\right)+\left[d_{1}-1=k\right] c\left(d_{2}\right)\right) t\right) \\
& \times \frac{\left(d_{1}-1\right)+k(a-1)}{(a+1)(k t+i-1)-1} \\
& +\left(\tilde{r}_{2}\left(d_{1}, d_{2}-1, t, i-1\right)+\frac{i-2}{k}\left(\left[d_{2}-1=k\right] c\left(d_{1}\right)+\left[d_{1}=k\right] c\left(d_{2}-1\right)\right) t\right) \\
& \times \frac{\left(d_{2}-1\right)+k(a-1)}{(a+1)(k t+i-1)-1} \\
& +\left(\tilde{r}_{2}\left(d_{1}, d_{2}, t, i-1\right)+\frac{i-2}{k}\left(\left[d_{2}=k\right] c\left(d_{1}\right)+\left[d_{1}=k\right] c\left(d_{2}\right)\right) t\right) \\
& \left(1-\frac{d_{1}+d_{2}+2 k(a-1)}{(a+1)(k t+i-1)-1}\right)+\left[d_{1} \leq k+1\right] O\left(c\left(d_{2}\right)\right) \\
& +\left[d_{2} \leq k+1\right] O\left(c\left(d_{1}\right)\right) \text {. }
\end{aligned}
$$

The assumptions $d_{1}+d_{2} \leq k t+2 k+(i-1)$ and $t>2+1 / k a$ imply that

$$
1-\frac{d_{1}+d_{2}+2 k(a-1)}{(a+1)(k t+i-1)-1} \geq 0
$$


By the induction hypothesis,

$$
\begin{aligned}
& \left|\tilde{r}_{2}\left(d_{1}, d_{2}, t, i\right)+\frac{i-1}{k}\left(\left[d_{2}=k\right] c\left(d_{1}\right)+\left[d_{1}=k\right] c\left(d_{2}\right)\right) t\right| \\
& \leq C\left(k t+\frac{a+\frac{1}{2}}{a+1}(i-1)\right) \\
& \quad \times\left(\left(c_{1}\left(d_{1}-1, d_{2}\right)+c_{1}\left(d_{2}, d_{1}-1\right)\right) \frac{\left(d_{1}-1\right)+k(a-1)}{(a+1)(k t+i-1)-1}\right. \\
& \quad+\left(c_{1}\left(d_{1}, d_{2}-1\right)+c_{1}\left(d_{2}-1, d_{1}\right)\right) \frac{\left(d_{2}-1\right)+k(a-1)}{(a+1)(k t+i-1)-1} \\
& \left.\quad+\left(c_{1}\left(d_{1}, d_{2}\right)+c_{1}\left(d_{2}, d_{1}\right)\right)\left(1-\frac{d_{1}+d_{2}+2 k(a-1)}{(a+1)(k t+i-1)-1}\right)\right) \\
& +\left[d_{1} \leq k+1\right] O\left(c\left(d_{2}\right)\right)+\left[d_{2} \leq k+1\right] O\left(c\left(d_{1}\right)\right) .
\end{aligned}
$$

Since

$$
\begin{aligned}
& \frac{c_{1}\left(d_{1}-1, d_{2}\right)}{c_{1}\left(d_{1}, d_{2}\right)} \frac{\left(d_{1}-1\right)+k(a-1)}{(a+1)(k t+i-1)-1}+\frac{c_{1}\left(d_{1}, d_{2}-1\right)}{c_{1}\left(d_{1}, d_{2}\right)} \frac{\left(d_{2}-1\right)+k(a-1)}{(a+1)(k t+i-1)-1} \\
& \quad+1-\frac{d_{1}+d_{2}+2 k(a-1)}{(a+1)(k t+i-1)-1} \\
& =\left(1-\left[d_{1}=k\right]\right) \frac{d_{1}-k+k a+a}{(a+1)(k t+i-1)-1} \\
& \quad+\left(1-\left[d_{2}=k\right]\right) \frac{d_{2}+k(a-1)}{(a+1)(k t+i-1)-1}+1-\frac{d_{1}+d_{2}+2 k(a-1)}{(a+1)(k t+i-1)-1} \\
& \leq 1+\frac{a}{(a+1)(k t+i-1)-1},
\end{aligned}
$$

we have

$$
\begin{aligned}
&\left|\tilde{r}_{2}\left(d_{1}, d_{2}, t, i\right)+\frac{i-1}{k}\left(\left[d_{2}=k\right] c\left(d_{1}\right)+\left[d_{1}=k\right] c\left(d_{2}\right)\right) t\right| \\
& \leq C\left(c_{1}\left(d_{1}, d_{2}\right)+c_{1}\left(d_{2}, d_{1}\right)\right)\left(k t+\frac{a+\frac{1}{2}}{a+1}(i-1)\right) \\
& \times\left(1+\frac{a}{(a+1)(k t+i-1)-1}\right) \\
&+\left[d_{1} \leq k+1\right] O\left(c\left(d_{2}\right)\right)+\left[d_{2} \leq k+1\right] O\left(c\left(d_{1}\right)\right) .
\end{aligned}
$$


Since

$$
\begin{aligned}
(k t & \left.+\frac{a+\frac{1}{2}}{a+1} i\right)-\left(k t+\frac{a+\frac{1}{2}}{a+1}(i-1)\right)\left(1+\frac{a}{(a+1)(k t+i-1)-1}\right) \\
= & \frac{a+\frac{1}{2}}{a+1}-\left(k t+\frac{a+\frac{1}{2}}{a+1}(i-1)\right) \frac{a}{(a+1)(k t+i-1)-1} \\
= & \frac{1}{(a+1)(k t+i-1)-1} \\
& \times\left(\left(a+\frac{1}{2}\right)(k t+i-1)-\frac{a+\frac{1}{2}}{a+1}-a k t-a \frac{a+\frac{1}{2}}{a+1}(i-1)\right) \\
= & \frac{1}{(a+1)(k t+i-1)-1}\left(\frac{k t}{2}+\frac{a+\frac{1}{2}}{a+1}(i-2)\right)
\end{aligned}
$$

is always positive and tends to a nonzero constant limit as $t$ grows, it is bounded from below by a positive constant. Therefore, for all sufficiently large values of $C$, the inequality (4.7) implies the inductive step by $i$, and so (4.6) holds.

As a consequence of (4.6), we obtain

$$
\tilde{r}_{2}\left(d_{1}, d_{2}, t, i\right)=O\left(\frac{t}{d_{1}^{a+1} d_{2}}+\frac{t}{d_{1} d_{2}^{a+1}}\right) .
$$

The proven bound, the representation (4.1), and Theorem 2.1 give the following bound:

$$
\begin{aligned}
& \operatorname{cov}\left(R\left(d_{1}, t\right), R\left(d_{2}, t\right)\right) \\
& \quad=O\left(\frac{t}{d_{1}^{a+1} d_{2}}+\frac{t}{d_{1} d_{2}^{a+1}}\right)+O\left(d_{1}^{-2-a} t\right)+O\left(d_{2}^{-2-a} t\right)+O\left(\frac{1}{d_{1} d_{2}}\right) .
\end{aligned}
$$

If $d_{1} \leq d_{2}$, the maximum among the first three terms on the right-hand side is $O\left(d_{1}^{-2-a} t\right)$; otherwise, the maximum is $O\left(d_{2}^{-2-a} t\right)$. This proves Theorem 2.2.

\section{Proof of Theorem 2.4}

We will use the notation $N\left(s_{1}, s_{2}\right)$ for the number of edges between nodes $s_{1}$ and $s_{2}$. As usual, $N_{t, i}\left(s_{1}, s_{2}\right)$ is the value of $N\left(s_{1}, s_{2}\right)$ in the graph before the $i$ th step.

First, we define a function

$$
f\left(d_{1}, d_{2}, t, i\right)=E_{t, i}\left(\sum_{s_{1}=1}^{t} \sum_{\substack{s_{2}=1 \\ s_{2} \neq s_{1}}}^{t}\left[\operatorname{deg} s_{1}=d_{1}, \operatorname{deg} s_{2}=d_{2}\right] N\left(s_{1}, s_{2}\right)\right) .
$$

It is easy to see that $E X\left(d_{1}, d_{2}, t\right)=f\left(d_{1}, d_{2}, t, 1\right)$. 
Recurrent equations on $f$ are deduced as was done in the previous sections. The sum (5.1) does not include the last node, so $N\left(s_{1}, s_{2}\right)$ does not change when a new edge is added. Thus, the $i$ th step acts on $f$ as in the case of $r_{2}$ (compare with (4.2)):

$$
\begin{aligned}
f\left(d_{1}, d_{2}, t, i+1\right)= & f\left(d_{1}-1, d_{2}, t, i\right) \frac{\left(d_{1}-1\right)+k(a-1)}{(a+1)(k t+i)-1} \\
& +f\left(d_{1}, d_{2}-1, t, i\right) \frac{\left(d_{2}-1\right)+k(a-1)}{(a+1)(k t+i)-1} \\
& +f\left(d_{1}, d_{2}, t, i\right)\left(1-\frac{d_{1}+d_{2}+2 k(a-1)}{(a+1)(k t+i)-1}\right) .
\end{aligned}
$$

Second, we define a function

$$
g\left(d_{1}, d_{2}, t, i\right)=E_{t, i}\left(\left[\operatorname{deg}(t+1)=d_{2}\right] \sum_{s=1}^{t}\left[\operatorname{deg} s=d_{1}\right] N(t+1, s)\right) .
$$

Obviously,

$$
f\left(d_{1}, d_{2}, t+1,1\right)=f\left(d_{1}, d_{2}, t, k+1\right)+g\left(d_{1}, d_{2}, t, k+1\right)+g\left(d_{2}, d_{1}, t, k+1\right),
$$

and since $N(t+1, s)=0$ before any edges from the node $t+1$ are added, we have

$$
g\left(d_{1}, d_{2}, t, 1\right)=0
$$

We now consider one summand of the sum (5.3) and the $i$ th step. Let the new edge link nodes be $t+1$ and $\gamma$. We have three nonintersecting cases: $\gamma=s$, $\gamma=t+1, \gamma \notin\{s, t+1\}$. Note that

$$
\begin{aligned}
& {\left[\gamma=s, \operatorname{deg}_{t, i+1}(t+1)=d_{2}, \operatorname{deg}_{t, i+1} s=d_{1}\right] N_{t, i+1}(s)} \\
& \quad=\left[\gamma=s, \operatorname{deg}_{t, i}(t+1)=d_{2}-1, \operatorname{deg}_{t, i} s=d_{1}-1\right]\left(N_{t, i}(s)+1\right), \\
& \quad\left[\gamma=t+1, \operatorname{deg}_{t, i+1}(t+1)=d_{2}, \operatorname{deg}_{t, i+1} s=d_{1}\right] N_{t, i+1}(s) \\
& \quad=\left[\gamma=t+1, \operatorname{deg}_{t, i}(t+1)=d_{2}-2, \operatorname{deg}_{t, i} s=d_{1}\right] N_{t, i}(s), \\
& {\left[\gamma \notin\{s, t+1\}, \operatorname{deg}_{t, i+1}(t+1)=d_{2}, \operatorname{deg}_{t, i+1} s=d_{1}\right] N_{t, i+1}(s)} \\
& \quad=\left[\gamma \notin\{s, t+1\}, \operatorname{deg}_{t, i}(t+1)=d_{2}-1, \operatorname{deg}_{t, i} s=d_{1}\right] N_{t, i}(s) .
\end{aligned}
$$


Taking the expectation and using the definition (4.4), we obtain

$$
\begin{aligned}
& g\left(d_{1}, d_{2}, t, i+1\right) \\
& \quad=\left(g\left(d_{1}-1, d_{2}-1, t, i\right)+r_{2}^{\prime}\left(d_{1}-1, d_{2}-1, t, i\right)\right) \frac{\left(d_{1}-1\right)+k(a-1)}{(a+1)(k t+i)-1} \\
& \quad+g\left(d_{1}, d_{2}-2, t, i\right) \frac{\left(d_{2}-2\right)+(i-1)(a-1)+a}{(a+1)(k t+i)-1} \\
& \quad+g\left(d_{1}, d_{2}-1, t, i\right)\left(1-\frac{\left(d_{1}-1\right)+k(a-1)+\left(d_{2}-1\right)+(i-1)(a-1)+a}{(a+1)(k t+i)-1}\right) .
\end{aligned}
$$

Third, we derive a bound on $g$. Obviously, $g\left(d_{1}, d_{2}, t, i\right)=0$ when $d_{2}>2(i-1)$ or $d_{1} \geq 2(k t+i)$. If $d_{1}<2(k t+i)$ and $d_{2} \leq 2(i-1)$, then

$$
\frac{d_{1}+d_{2}}{t} \cdot O\left(d_{1}^{-a-1}\right)=O\left(d_{1}^{-a} t^{-1}\right)=O\left(t^{-1}\right) \quad \text { and } \quad \frac{d_{1}+d_{2}}{t} \cdot O\left(t^{-1}\right)=O\left(t^{-1}\right) \text {. }
$$

Recall that we have proved the bound (4.5) on $r_{2}^{\prime}$. It is easy to see now that

$$
g\left(d_{1}, d_{2}, t, i+1\right)=i\left[d_{2}=i\right] c\left(d_{1}-1\right) \frac{\left(d_{1}-1\right)+k(a-1)}{(a+1) k}+O\left(\frac{1}{t}\right) .
$$

Finally, we are ready to study $f$. For the rest of the proof, we will assume that $d_{1} \geq k$ and $d_{2} \geq k$.

We set

$$
A=\frac{\Gamma(a+2)}{\mathrm{B}(k a, a+1)}=(a+1) \frac{\Gamma(k a+a+1)}{\Gamma(k a)},
$$

$D_{1}=d_{1}-k+k a$, and $D_{2}=d_{2}-k+k a$ for brevity. By definition,

$$
c\left(d_{1}\right)=A \frac{\Gamma\left(D_{1}\right)}{\Gamma\left(D_{1}+a+2\right)} .
$$

Let $c_{X}\left(d_{1}, d_{2}\right)$ be defined recurrently as follows:

$$
\begin{aligned}
c_{X}(k, k) & =0, \\
c_{X}\left(d_{1}, k\right) & =\frac{\left(D_{1}-1\right)\left(c_{X}\left(d_{1}-1, k\right)+c\left(d_{1}-1\right)\right)}{D_{1}+k a+a+1}, \quad d_{1}>k, \\
c_{X}\left(k, d_{2}\right) & =\frac{\left(D_{2}-1\right)\left(c_{X}\left(k, d_{2}-1\right)+c\left(d_{2}-1\right)\right)}{D_{2}+k a+a+1}, \quad d_{2}>k, \\
c_{X}\left(d_{1}, d_{2}\right) & =\frac{\left(D_{1}-1\right) c_{X}\left(d_{1}-1, d_{2}\right)+\left(D_{2}-1\right) c_{X}\left(d_{1}, d_{2}-1\right)}{D_{1}+D_{2}+a+1}, \quad d_{1}, d_{2}>k .
\end{aligned}
$$

Let

$$
\begin{aligned}
& c_{2}\left(d_{1}, d_{2}\right)=\frac{\Gamma\left(D_{1}\right) \Gamma\left(D_{2}\right) \Gamma\left(D_{1}+D_{2}+3\right)}{\Gamma\left(D_{1}+2\right) \Gamma\left(D_{2}+2\right) \Gamma\left(D_{1}+D_{2}+a+2\right)}, \\
& c_{3}\left(d_{1}, d_{2}\right)=\frac{\Gamma\left(D_{1}\right) \Gamma\left(D_{2}\right) \Gamma\left(D_{1}+D_{2}+1\right)}{\Gamma\left(D_{1}+1\right) \Gamma\left(D_{2}+1\right) \Gamma\left(D_{1}+D_{2}+a+2\right)} .
\end{aligned}
$$


Obviously, these functions are symmetric. If $d_{1}>k$, then

$$
\begin{aligned}
& \frac{c_{2}\left(d_{1}-1, d_{2}\right)}{c_{2}\left(d_{1}, d_{2}\right)}=\frac{\left(D_{1}+1\right)\left(D_{1}+D_{2}+a+1\right)}{\left(D_{1}-1\right)\left(D_{1}+D_{2}+2\right)}, \\
& \frac{c_{3}\left(d_{1}-1, d_{2}\right)}{c_{3}\left(d_{1}, d_{2}\right)}=\frac{D_{1}\left(D_{1}+D_{2}+a+1\right)}{\left(D_{1}-1\right)\left(D_{1}+D_{2}\right)} .
\end{aligned}
$$

Thus, for $d_{1}, d_{2}>k$,

$$
\begin{aligned}
& c_{2}\left(d_{1}, d_{2}\right)=\frac{\left(D_{1}-1\right) c_{2}\left(d_{1}-1, d_{2}\right)+\left(D_{2}-1\right) c_{2}\left(d_{1}, d_{2}-1\right)}{D_{1}+D_{2}+a+1}, \\
& c_{3}\left(d_{1}, d_{2}\right)=\frac{\left(D_{1}-1\right) c_{3}\left(d_{1}-1, d_{2}\right)+\left(D_{2}-1\right) c_{3}\left(d_{1}, d_{2}-1\right)}{D_{1}+D_{2}+a+1}
\end{aligned}
$$

and

$$
\begin{aligned}
\frac{c\left(d_{1}\right)}{A k a c_{2}\left(d_{1}, k\right)} & =\frac{\Gamma\left(D_{1}+2\right) \Gamma(k a+2) \Gamma\left(D_{1}+k a+a+2\right)}{k a \Gamma\left(D_{1}+a+2\right) \Gamma(k a) \Gamma\left(D_{1}+k a+3\right)} \\
& =\frac{k a+1}{D_{1}+k a+2} \frac{\Gamma\left(D_{1}+2\right) \Gamma\left(D_{1}+k a+a+2\right)}{\Gamma\left(D_{1}+a+2\right) \Gamma\left(D_{1}+k a+2\right)}
\end{aligned}
$$

Let $(\alpha)_{n}=\alpha(\alpha+1) \cdots(\alpha+n-1)$ be the Pochhammer symbol. Let

$$
{ }_{2} F_{1}(\alpha, \beta ; \gamma ; z)=\sum_{n=0}^{\infty} \frac{(\alpha)_{n}(\beta)_{n}}{(\gamma)_{n}} \frac{z^{n}}{n !}
$$

for $\gamma \neq 0,-1,-2, \ldots$ be the hypergeometric function. According to [Abramowitz and Stegun 64, 15.1.1], if $\gamma-\alpha-\beta>0$ and $|z| \leq 1$, this series converges absolutely. We quote the following formula from [Abramowitz and Stegun 64, 15.1.20]:

$$
{ }_{2} F_{1}(\alpha, \beta ; \gamma ; 1)=\frac{\Gamma(\gamma) \Gamma(\gamma-\alpha-\beta)}{\Gamma(\gamma-\alpha) \Gamma(\gamma-\beta)} .
$$

Thus

$$
\begin{aligned}
\frac{\Gamma\left(D_{1}+2\right) \Gamma\left(D_{1}+k a+a+2\right)}{\Gamma\left(D_{1}+a+2\right) \Gamma\left(D_{1}+k a+2\right)} & ={ }_{2} F_{1}\left(a, k a ; D_{1}+k a+a+2 ; 1\right) \\
& =\sum_{n=0}^{\infty} \frac{(a)_{n}(k a)_{n}}{\left(D_{1}+k a+a+2\right)_{n} n !} .
\end{aligned}
$$

Since all terms of the last series are positive and the first term is 1 , it follows that

$$
\frac{c\left(d_{1}\right)}{A k a c_{2}\left(d_{1}, k\right)} \geq \frac{k a+1}{D_{1}+k a+2} .
$$


Moreover,

$$
\begin{aligned}
& \frac{\Gamma\left(D_{1}+2\right) \Gamma\left(D_{1}+k a+a+2\right)}{\Gamma\left(D_{1}+a+2\right) \Gamma\left(D_{1}+k a+2\right)} \\
& \quad=1+\sum_{n=0}^{\infty} \frac{(a)_{n+1}(k a)_{n+1}}{\left(D_{1}+k a+a+2\right)_{n+1}(n+1) !} \\
& \quad=1+\frac{a^{2} k}{D_{1}+k a+a+2} \sum_{n=0}^{\infty} \frac{(a+1)_{n}(k a+1)_{n}}{\left(D_{1}+k a+a+3\right)_{n}(n+1) !} \\
& \quad \leq 1+\frac{a^{2} k}{D_{1}+k a+a+2}{ }_{2} F_{1}(a+1, k a+1 ; 2 k a+a+3 ; 1) \\
& \quad \leq 1+\frac{a^{2} k}{D_{1}+k a+1} \frac{\Gamma(k a+1) \Gamma(2 k a+a+3)}{\Gamma(2 k a+2) \Gamma(k a+a+2)},
\end{aligned}
$$

and also

$$
\begin{aligned}
\frac{c\left(d_{1}\right)}{A k a c_{2}\left(d_{1}, k\right)} & \leq \frac{k a+1}{D_{1}+k a+2}\left(1+\frac{k a B}{D_{1}+k a+1}\right), \\
B & =a \frac{\Gamma(k a+1) \Gamma(2 k a+a+3)}{\Gamma(2 k a+2) \Gamma(k a+a+2)} .
\end{aligned}
$$

In Theorem 2.4, we have three assertions. The first one says that

$$
E X\left(d_{1}, d_{2}, t\right)=c_{X}\left(d_{1}, d_{2}\right) t+O_{a, k}(1)
$$

The second one gives a bound for $c_{X}$. The third one gives an asymptotic formula for $c_{X}$. Now we shall show that our function $c_{X}$ admits the bound from the second assertion. This bound is equivalent to

$$
\begin{aligned}
& A k a\left(c_{2}\left(d_{1}, d_{2}\right)-\left(4-\frac{2}{1+k a}\right) c_{3}\left(d_{1}, d_{2}\right)\right) \\
& \quad \leq c_{X}\left(d_{1}, d_{2}\right) \leq A k a\left(c_{2}\left(d_{1}, d_{2}\right)+B c_{3}\left(d_{1}, d_{2}\right)\right) .
\end{aligned}
$$

To prove (5.8), we use induction on $d_{1}+d_{2}$. If $d_{1}=d_{2}=k$, the right-hand side of the inequality is obvious, and its left-hand side follows from

$$
\frac{c_{2}(k, k)}{c_{3}(k, k)}=\frac{(2 k a+2)(2 k a+1)}{(k a+1)(k a+1)}=\frac{4 k a+2}{k a+1}=4-\frac{2}{k a+1} .
$$

If $d_{1}>k$ and $d_{2}>k$, all the parts of (5.8) satisfy the same recurrent equation, so (5.8) follows from the induction hypothesis. Due to symmetry, it remains to 
prove (5.8) for $d_{2}=k, d_{1}>k$. We have

$$
\begin{aligned}
\frac{k a\left(c_{2}\left(d_{1}, k\right)-\frac{2+4 k a}{1+k a} c_{3}\left(d_{1}, k\right)\right)}{k a c_{2}\left(d_{1}, k\right)} & =1-\frac{\left(D_{1}+1\right)(2+4 k a)}{\left(D_{1}+k a+2\right)\left(D_{1}+k a+1\right)} \\
& =\frac{\left(D_{1}-k a\right)\left(D_{1}-k a+1\right)}{\left(D_{1}+k a+1\right)\left(D_{1}+k a+2\right)} .
\end{aligned}
$$

In particular,

$$
k a\left(c_{2}\left(d_{1}, k\right)-\frac{2+4 k a}{1+k a} c_{3}\left(d_{1}, k\right)\right)>0
$$

for $d_{1}>k$. Then

$$
\begin{aligned}
& \frac{c_{X}\left(d_{1}, k\right)}{A k a\left(c_{2}\left(d_{1}, k\right)-\frac{2+4 k a}{1+k a} c_{3}\left(d_{1}, k\right)\right)} \\
= & \frac{\left(D_{1}+k a+1\right)\left(D_{1}+1\right)\left(D_{1}+k a+a+1\right)}{\left(D_{1}-k a\right)\left(D_{1}-k a+1\right)\left(D_{1}-1\right)} \frac{c_{X}\left(d_{1}, k\right)}{A k a c_{2}\left(d_{1}-1, k\right)} \\
= & \frac{\left(D_{1}+k a+1\right)\left(D_{1}+1\right)}{\left(D_{1}-k a\right)\left(D_{1}-k a+1\right)} \frac{c_{X}\left(d_{1}-1, k\right)+c\left(d_{1}-1\right)}{A k a c_{2}\left(d_{1}-1, k\right)} \\
\geq & \frac{\left(D_{1}+k a+1\right)\left(D_{1}+1\right)}{\left(D_{1}-k a\right)\left(D_{1}-k a+1\right)}\left(\frac{\left(D_{1}-1-k a\right)\left(D_{1}-k a\right)}{\left(D_{1}+k a\right)\left(D_{1}+k a+1\right)}+\frac{k a+1}{D_{1}+k a+1}\right) \\
= & \frac{D_{1}+1}{D_{1}-k a+1}\left(\frac{D_{1}-1-k a}{D_{1}+k a}+\frac{k a+1}{D_{1}-k a}\right) .
\end{aligned}
$$

Furthermore,

$$
\begin{aligned}
& \frac{D_{1}-1-k a}{D_{1}+k a}+\frac{k a+1}{D_{1}-k a}-\frac{D_{1}-k a+1}{D_{1}+1} \\
& =\frac{k a+1}{D_{1}-k a}-\frac{(k a+1) D_{1}-k^{2} a^{2}+2 k a+1}{\left(D_{1}+1\right)\left(D_{1}+k a\right)} \\
& \geq(k a+1)\left(\frac{1}{D_{1}-k a}-\frac{D_{1}+k a+1}{\left(D_{1}+1\right)\left(D_{1}+k a\right)}\right) \\
& =(k a+1) k a \frac{D_{1}+k a+2}{\left(D_{1}+1\right)\left(D_{1}+k a\right)\left(D_{1}-k a\right)}>0 .
\end{aligned}
$$

This proves the left-hand side of inequality (5.8). 
Now,

$$
\begin{aligned}
\frac{c_{X}\left(d_{1}, k\right)}{A k a\left(c_{2}\left(d_{1}, k\right)+B c_{3}\left(d_{1}, k\right)\right)} \\
=\left(1+B \frac{\left(D_{1}+1\right)(k a+1)}{\left(D_{1}+k a+1\right)\left(D_{1}+k a+2\right)}\right)^{-1} \frac{\left(D_{1}+1\right)\left(D_{1}+k a+a+1\right)}{\left(D_{1}-1\right)\left(D_{1}+k a+2\right)} \\
\quad \times \frac{c_{X}\left(d_{1}, k\right)}{A k a c_{2}\left(d_{1}-1, k\right)} \\
\leq \frac{\left(D_{1}+k a+1\right)\left(D_{1}+1\right)}{\left(D_{1}+k a+1\right)\left(D_{1}+k a+2\right)+B\left(D_{1}+1\right)(k a+1)} \\
\quad \times\left(1+B \frac{D_{1}(k a+1)}{\left(D_{1}+k a\right)\left(D_{1}+k a+1\right)}+\frac{k a+1}{D_{1}+k a+1}\left(1+\frac{k a B}{D_{1}+k a}\right)\right) .
\end{aligned}
$$

Furthermore,

$$
\begin{aligned}
(1+ & \left.B \frac{D_{1}(k a+1)}{\left(D_{1}+k a\right)\left(D_{1}+k a+1\right)}+\frac{k a+1}{D_{1}+k a+1}\left(1+\frac{k a B}{D_{1}+k a}\right)\right) \\
& -\left(\frac{D_{1}+k a+2}{D_{1}+1}+B \frac{k a+1}{D_{1}+k a+1}\right) \\
= & \left(\frac{k a+1}{D_{1}+k a+1}-\frac{k a+1}{D_{1}+1}\right)<0 .
\end{aligned}
$$

This proves the right-hand side of inequality (5.8).

The third assertion of Theorem 2.4 (i.e., the asymptotic formula for $c_{X}\left(d_{1}, d_{2}\right)$ ) is derived from (5.8) in the same way as a similar formula was derived from (3.6).

It remains to prove the first assertion. We will use the bound

$$
c_{X}\left(d_{1}, d_{2}\right)=O\left(\frac{\left(d_{1}+d_{2}\right)^{1-a}}{d_{1}^{2} d_{2}^{2}}\right) .
$$

Let

$$
\tilde{f}\left(d_{1}, d_{2}, t, i\right)=f\left(d_{1}, d_{2}, t, i\right)-c_{X}\left(d_{1}, d_{2}\right)\left(t+\frac{i}{k}-\frac{1}{k(a+1)}\right) .
$$

Let $C=C(a, k)$ be a sufficiently large constant that will be determined later.

We claim that

$$
\begin{aligned}
& \mid \tilde{f}\left(d_{1}, d_{2}, t, i\right) \\
& \quad+(i-1)\left(\left[d_{1}=k\right] \frac{\left(D_{2}-1\right) c\left(d_{2}-1\right)}{(a+1) k}+\left[d_{2}=k\right] \frac{\left(D_{1}-1\right) c\left(d_{1}-1\right)}{(a+1) k}\right) \mid \\
& \quad \leq C\left(1-\frac{1}{(a+2) k(t+1)}\right)^{i-1} .
\end{aligned}
$$


Since $r_{2}^{\prime}\left(d_{1}, d_{2}, t, i\right)=0$ when $d_{1}+d_{2}>k t+k+2(i-1),(5.5)$ and (5.6) imply that $g\left(d_{1}, d_{2}, t, i\right)=0$ when $d_{1}+d_{2}>k t+k+2(i-1)$. Consequently, (5.2) and (5.4) imply that $f\left(d_{1}, d_{2}, t, i\right)=0$ when $d_{1}+d_{2}>k t+2 k+(i-1)$.

If $d_{1}+d_{2}>k t+2 k+(i-1)$, then

$$
\tilde{f}\left(d_{1}, d_{2}, t, i\right)=-c_{X}\left(d_{1}, d_{2}\right)\left(t+\frac{i}{k}-\frac{1}{k(a+1)}\right)=O\left(\frac{\left(d_{1}+d_{2}\right)^{2-a}}{d_{1}^{2} d_{2}^{2}}\right),
$$

so (5.9) holds for all sufficiently large values of $C$.

Now assume $d_{1}+d_{2} \leq k t+2 k+(i-1)$. We will prove $(5.9)$ by induction on $t$ and, for fixed $t$, on $i$. The basis of induction $t=1, \ldots, 2+\lfloor 1 / k a\rfloor$ and any $i=1, \ldots, k+1$ obviously holds for all sufficiently large values of $C$.

Now let $t \geq 3+\lfloor 1 / k a\rfloor$ and let (5.9) hold for $t-1$. Since (5.9) is trivial for $d_{1}=d_{2}=k$ and symmetric, we may assume that $d_{1}>k$. From (5.4) and (5.7), we obtain

$$
\begin{aligned}
\left|\tilde{f}\left(d_{1}, d_{2}, t, 1\right)\right| & =\left|\tilde{f}\left(d_{1}, d_{2}, t-1, k+1\right)+k\left[d_{2}=k\right] c\left(d_{1}-1\right) \frac{D_{1}-1}{(a+1) k}+O\left(\frac{1}{t}\right)\right| \\
& \leq C\left(1-\frac{1}{(a+2) k t}\right)^{k}+O\left(\frac{1}{t}\right) .
\end{aligned}
$$

The right-hand side is less than $C$ for all sufficiently large values of $C$.

Finally, let

$$
t \geq 3+\left\lfloor\frac{1}{k a}\right\rfloor>2+\frac{1}{k a}, \quad i>1
$$

and let (5.9) hold for $i-1$. We reuse the notation $T=(a+1)(k t+i-1)-1$ and again assume $d_{1}>k$. From (5.2) we obtain

$$
\begin{gathered}
\tilde{f}\left(d_{1}-1, d_{2}, t, i-1\right) \frac{D_{1}-1}{T}+\left[d_{2}>k\right] \tilde{f}\left(d_{1}, d_{2}-1, t, i-1\right) \frac{D_{2}-1}{T} \\
+\tilde{f}\left(d_{1}, d_{2}, t, i-1\right)\left(1-\frac{D_{1}+D_{2}}{T}\right) \\
=\tilde{f}\left(d_{1}, d_{2}, t, i\right)+c_{X}\left(d_{1}, d_{2}\right)\left(\frac{T}{(a+1) k}+\frac{1}{k}\right) \\
\quad-\frac{1}{(a+1) k}\left(c_{X}\left(d_{1}-1, d_{2}\right)\left(D_{1}-1\right)+\left[d_{2}>k\right] c_{X}\left(d_{1}, d_{2}-1\right)\left(D_{2}-1\right)\right. \\
\left.\quad+c_{X}\left(d_{1}, d_{2}\right)\left(T-\left(D_{1}+D_{2}\right)\right)\right) \\
=\tilde{f}\left(d_{1}, d_{2}, t, i\right)+\left[d_{2}=k\right] c\left(d_{1}-1\right) \frac{D_{1}-1}{(a+1) k} .
\end{gathered}
$$


The assumptions $d_{1}+d_{2} \leq k t+2 k+(i-1)$ and $t>2+1 / k a$ imply that

$$
1-\frac{D_{1}+D_{2}}{T}=1-\frac{d_{1}+d_{2}+2 k(a-1)}{(a+1)(k t+i-1)-1} \geq 0 .
$$

Since $\left(D_{1}-2\right) c\left(d_{1}-2\right)=\left[d_{1}>k+1\right]\left(D_{1}+a\right) c\left(d_{1}-1\right)$, we have

$$
\begin{aligned}
(i-2)\left[d_{2}=k\right] \frac{\left(D_{1}-2\right) c\left(d_{1}-2\right)}{(a+1) k} \frac{D_{1}-1}{T} \\
\quad+(i-2)\left[d_{2}=k\right] \frac{\left(D_{1}-1\right) c\left(d_{1}-1\right)}{(a+1) k}\left(1-\frac{D_{1}+D_{2}}{T}\right) \\
=(i-2)\left[d_{2}=k\right] \frac{\left(D_{1}-1\right) c\left(d_{1}-1\right)}{(a+1) k}\left(\left[d_{1}>k+1\right] \frac{D_{1}+a}{T}+1-\frac{D_{1}+D_{2}}{T}\right) \\
=(i-2)\left[d_{2}=k\right] \frac{\left(D_{1}-1\right) c\left(d_{1}-1\right)}{(a+1) k}+O\left(\frac{1}{t}\right) .
\end{aligned}
$$

If $d_{2}>k$, then

$$
\frac{D_{1}-1}{T}+\frac{D_{2}-1}{T}+1-\frac{D_{1}+D_{2}}{T}=1-\frac{2}{T} \leq 1-\frac{1}{T}
$$

If $d_{2}=k$, then

$$
\frac{D_{1}-1}{T}+1-\frac{D_{1}+D_{2}}{T}=1-\frac{k a+1}{T} \leq 1-\frac{1}{T} .
$$

Thus

$$
\begin{aligned}
& \left|\tilde{f}\left(d_{1}, d_{2}, t, i\right)+(i-1)\left[d_{2}=k\right] \frac{\left(D_{1}-1\right) c\left(d_{1}-1\right)}{(a+1) k}\right| \\
& \quad \leq C\left(1-\frac{1}{(a+2) k(t+1)}\right)^{i-2}\left(1-\frac{1}{(a+1) k(t+1)}\right)+O\left(\frac{1}{t}\right) .
\end{aligned}
$$

For all sufficiently large values of $C$, the right-hand side is less than

$$
C\left(1-\frac{1}{(a+2) k(t+1)}\right)^{i-1}
$$

so the induction on $i$ is complete.

Theorem 2.4 follows from the proven bound (5.9).

\section{Proof of Theorem 2.5}

We use the Azuma-Hoeffding inequality. 
Theorem 6.I. [Azuma 67, Hoeffding 63] Let $\left(X_{s}\right)_{s=0}^{n}$ be a martingale with $\mid X_{s+1}-$ $X_{s} \mid \leq \delta$ for $s=0, \ldots, n-1$ and $x>0$. Then

$$
P\left(\left|X_{n}-X_{0}\right| \geq x\right) \leq 2 \exp \left(-\frac{x^{2}}{2 \delta^{2} n}\right) .
$$

We fix $d_{1}, d_{2}, t$ and set $X=X\left(d_{1}, d_{2}, t\right)$. Let $G$ be a random graph in $H_{a, k}^{(t)}$; it has $k t$ edges, sorted by the creation time. Let $G^{(s)}$ be a graph with $s$ first edges. Let $X_{s}=E\left(X \mid G^{(s)}\right), s=0, \ldots, k t$. In this sequence, $X_{0}=E X, X_{k t}=X$. By definition of the probabilistic space, the sequence $X_{s}$ is a martingale. We will estimate possible differences between adjacent elements of the sequence.

We fix an arbitrary $s$ from 0 to $k t-1$. Let $v$ be the head of the last edge in $G^{(s+1)}$, so $v$ is a random quantity depending on $G$. By definition,

$$
\begin{aligned}
X_{s} & =\sum_{\gamma} \operatorname{Pr}(v=\gamma) E\left(X \mid G^{(s)}, v=\gamma\right), \\
X_{s+1} & =E\left(X \mid G^{(s)}, v=v\left(G^{(s+1)}\right)\right),
\end{aligned}
$$

where the sum is over all nodes of $G$. Hence it is clear that

$$
\begin{aligned}
\min _{\gamma} E\left(X \mid G^{(s)}, v=\gamma\right) & \leq X_{s}, X_{s+1} \leq \max _{\gamma} E\left(X \mid G^{(s)}, v=\gamma\right) \\
\left|X_{s}-X_{s+1}\right| & \leq \max _{\gamma} E\left(X \mid G^{(s)}, v=\gamma\right)-\min _{\gamma} E\left(X \mid G^{(s)}, v=\gamma\right) .
\end{aligned}
$$

Let $\gamma_{1} \in \arg \min E\left(X \mid G^{(s)}, v=\gamma\right)$ and $\gamma_{2} \in \arg \max E\left(X \mid G^{(s)}, v=\gamma\right)$. It is sufficient to prove an upper bound for

$$
E\left(X \mid G^{(s)}, v=\gamma_{2}\right)-E\left(X \mid G^{(s)}, v=\gamma_{1}\right) .
$$

We consider the sum

$$
X=\sum_{s_{1}=1}^{t} \sum_{\substack{s_{2}=1 \\ s_{2} \neq s_{1}}}^{t}\left[\operatorname{deg} s_{1}=d_{1}, \operatorname{deg} s_{2}=d_{2}\right] N\left(s_{1}, s_{2}\right) .
$$

Replacing the condition $v=\gamma_{1}$ by the condition $v=\gamma_{2}$ changes distributions of degrees of $\gamma_{i}$ and distributions of $N\left(\gamma_{i}, *\right)=N\left(*, \gamma_{i}\right)$; distributions of other values of $N$ do not change. Thus distributions of all terms in the sum (6.1) except those with $\left\{\gamma_{1}, \gamma_{2}\right\} \cap\left\{s_{1}, s_{2}\right\} \neq \varnothing$ are the same for $v=\gamma_{1}$ and $v=\gamma_{2}$. Let

$$
X^{\prime}=\sum_{s_{1}=1}^{t} \sum_{\substack{s_{2}=1 \\ s_{2} \neq s_{1} \\\left\{s_{1}, s_{2}\right\}\left\{\gamma_{1}, \gamma_{2}\right\} \neq \varnothing}}^{t}\left[\operatorname{deg} s_{1}=d_{1}, \operatorname{deg} s_{2}=d_{2}\right] N\left(s_{1}, s_{2}\right) .
$$


Then

$$
E\left(X-X^{\prime} \mid G^{(s)}, v=\gamma_{1}\right)=E\left(X-X^{\prime} \mid G^{(s)}, v=\gamma_{2}\right) .
$$

Obviously, $X^{\prime} \geq 0$. We have

$$
\begin{aligned}
& X^{\prime} \leq \sum_{s_{1}=1}^{t}\left[\operatorname{deg} s_{1}=d_{1}, \operatorname{deg} \gamma_{1}=d_{2}\right] N\left(s_{1}, \gamma_{1}\right) \\
& \quad+\sum_{s_{1}=1}^{t}\left[\operatorname{deg} s_{1}=d_{1}, \operatorname{deg} \gamma_{2}=d_{2}\right] N\left(s_{1}, \gamma_{2}\right) \\
& \quad+\sum_{s_{2}=1}^{t}\left[\operatorname{deg} \gamma_{1}=d_{1}, \operatorname{deg} s_{2}=d_{2}\right] N\left(\gamma_{1}, s_{2}\right) \\
& \quad+\sum_{s_{2}=1}^{t}\left[\operatorname{deg} \gamma_{2}=d_{1}, \operatorname{deg} s_{2}=d_{2}\right] N\left(\gamma_{2}, s_{2}\right) \\
& \leq\left[\operatorname{deg} \gamma_{1}=d_{2}\right] \sum_{s_{1}=1}^{t} N\left(s_{1}, \gamma_{1}\right)+\left[\operatorname{deg} \gamma_{2}=d_{2}\right] \sum_{s_{1}=1}^{t} N\left(s_{1}, \gamma_{2}\right) \\
& \quad+\left[\operatorname{deg} \gamma_{1}=d_{1}\right] \sum_{s_{2}=1}^{t} N\left(\gamma_{1}, s_{2}\right)+\left[\operatorname{deg} \gamma_{2}=d_{1}\right] \sum_{s_{2}=1}^{t} N\left(\gamma_{2}, s_{2}\right) \\
& =\left[\operatorname{deg} \gamma_{1}=d_{2}\right] d_{2}+\left[\operatorname{deg} \gamma_{2}=d_{2}\right] d_{2}+\left[\operatorname{deg} \gamma_{1}=d_{1}\right] d_{1}+\left[\operatorname{deg} \gamma_{2}=d_{1}\right] d_{1} \\
& \leq 2\left(d_{1}+d_{2}\right) .
\end{aligned}
$$

Thus,

$$
\begin{array}{r}
0 \leq E\left(X^{\prime} \mid G^{(s)}, v=\gamma_{1}\right), \quad E\left(X^{\prime} \mid G^{(s)}, v=\gamma_{2}\right) \leq 2\left(d_{1}+d_{2}\right) \\
\left|E\left(X^{\prime} \mid G^{(s)}, v=\gamma_{1}\right)-E\left(X^{\prime} \mid G^{(s)}, v=\gamma_{2}\right)\right| \leq 2\left(d_{1}+d_{2}\right) \\
\left|X_{s}-X_{s+1}\right| \leq 2\left(d_{1}+d_{2}\right)
\end{array}
$$

Consequently, the sequence $\left(X_{s}\right)$ satisfies the condition of Theorem 6.1 with $n=k t$ and $\delta=2\left(d_{1}+d_{2}\right)$. Substituting $x=c\left(d_{1}+d_{2}\right) \sqrt{k t}$ in Theorem 6.1 , we obtain Theorem 2.5.

\section{References}

[Abramowitz and Stegun 64] M. Abramowitz and I. A. Stegun (editors). Handbook of Mathematical Functions with Formulas, Graphs, and Mathematical Tables. Dover, 1964. 
[Azuma 67] K. Azuma. "Weighted Sums of Certain Dependent Variables." Tôhoku Math. J. 19 (1967), 357-367.

[Barabási and Albert 99] A.-L. Barabási and R. Albert. "Emergence of Scaling in Random Networks." Science 286 (1999), 509-512.

[Bollobás 01] B. Bollobás. Random Graphs. Cambridge Univ. Press, 2001.

[Bollobás and Riordan 03] B. Bollobás and O. M. Riordan. "Mathematical Results on Scale-Free Random Graphs." In Handbook of Graphs and Networks, pp. 1-34. Wiley$\mathrm{VCH}, 2003$.

[Bollobás et al. 01] B. Bollobás, O. M. Riordan, J. Spencer, and G. Tusnády. "The Degree Sequence of a Scale-Free Random Graph Process." Random Structures Algorithms 18:3 (2001), 279-290.

[Buckley and Osthus 04] P. G. Buckley and D. Osthus. "Popularity Based Random Graph Models Leading to a Scale-Free Degree Sequence." Discrete Math. 282 (2004), $53-68$.

[Dorogovtsev et al. 00] S. N. Dorogovtsev, J. F. F. Mendes, and A. N. Samukhin. "Structure of Growing Networks with Preferential Linking." Phys. Rev. Lett. 85 (2000), 4633.

[Drinea et al. 01] E. Drinea, M. Enachescu, and M. Mitzenmacher. "Variations on Random Graph Models for the Web." Technical report, Harvard University, Department of Computer Science, 2001.

[Durrett 07] R. Durrett. Random Graph Dynamics. Cambridge University Press, 2007.

[Janson et al. 00] S. Janson, T. Łuczak, and A. Ruciński. Random Graphs. Wiley, 2000.

[Jordan 06] J. Jordan. "The Degree Sequences and Spectra of Scale-Free Random Graphs." Random Structures and Algorithms 29 (2006), 226-242.

[Hoeffding 63] W. Hoeffding. "Probability Inequalities for Sums of Bounded Random Variables." J. Amer. Statist. Assoc. 58 (1963), 13-30.

Evgeniy A. Grechnikov, Yandex LLC, 16, Leo Tolstoy St., Moscow 119021, Russia (grechnik@mccme.ru) 